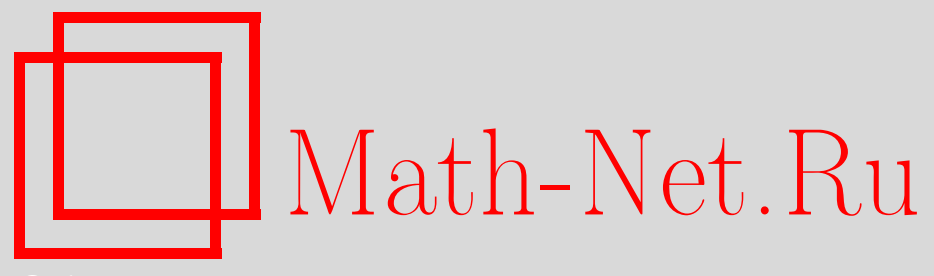

Дж. Харнад, А. Ю. Орлов, Фермионный подход к построению статистической суммы многоматричных моделей и многокомпонентной иерархии цепочки Тоды, TMФ, 2007, том 152, номер 2, 265-277

DOI: https://doi.org/10.4213/tmf6086

Использование Общероссийского математического портала Math-Net.Ru подразумевает, что вы прочитали и согласны с пользовательским соглашением http://www.mathnet.ru/rus/agreement

Параметры загрузки:

IP: 54.80 .73 .141

26 апреля 2023 г., 15:37:10

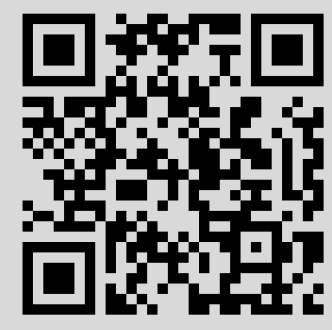




\title{
ФЕРМИОННЫЙ ПОДХОД К ПОСТРОЕНИЮ СТАТИСТИЧЕСКОЙ СУММЫ МНОГОМАТРИЧНЫХ МОДЕЛЕЙ И МНОГОКОМПОНЕНТНОЙ ИЕРАРХИИ ЦЕПОЧКИ ТОДЫ
}

\begin{abstract}
Для представления $(2 p-2) N$-кратных интегралов в виде фермионного вакуумного ожидания используются $p$-компонентные фермионы, $p=2,3, \ldots$ В результате получается фермионное представление для различных $(2 p-2)$-матричных моделей. Обсуждаются связи с $p$-компонетной иерархией Кадомцева-Петвиашвили, а также с иерархией $p$-компонентной цепочки Тоды. Показано, что множество всех, кроме двух, потоков $p$-компонентной цепочки Тоды переводит стандартные матричные модели в новые матричные модели.
\end{abstract}

Ключевые слова: матричные модели, тау-функция многокомпонентной цепочки Тоды, интегрируемые системы.

\section{1. ВВЕДЕНИЕ}

Пусть $d \mu_{\alpha}(x, y), \alpha=1, \ldots, p-1,-$ множество мер (вообще говоря, комплексных) с носителем на конечном множестве произведений кривых в комплексных плоскостях переменных $x$ и $y$; пусть также $\rho_{\alpha}, \alpha=2, \ldots, p-1,-$ множество функций от двух переменных; $x^{(\alpha)}=\left(x_{1}^{(\alpha)}, \ldots, x_{N}^{(\alpha)}\right), y^{(\alpha)}=\left(y_{1}^{(\alpha)}, \ldots, y_{N}^{(\alpha)}\right), \alpha=2, \ldots, p-1,-$ два набора переменных, а $x^{(p)}$ и $y^{(1)}$ задаются равенствами

$$
x_{i}^{(p)}=y_{i}^{(1)}=N-i, \quad i=1, \ldots, N .
$$

Мы будем использовать следующее обозначение:

$$
d \mu_{\alpha}\left(x^{(\alpha)}, y^{(\alpha+1)}\right):=\prod_{i=1}^{N} d \mu_{\alpha}\left(x_{i}^{(\alpha)}, y_{i}^{(\alpha+1)}\right) .
$$

${ }^{*}$ Centre de recherches mathématiques, Université de Montréal C. P. 6128, succ. centre ville, Montréal, Québec, Canada H3C 3J7. Department of Mathematics and Statistics, Concordia University 7141 Sherbrooke W., Montréal, Québec, Canada H4B 1R6.

E-mail: harnad@crm.umontreal.ca

$\dagger$ Лаборатория нелинейных волн, Институт океанологии РАН. E-mail: orlovs@wave.sio.rssi.ru 
Рассмотрим следующий $(2 p-2) N$-кратный интеграл по переменным $x^{(\alpha)}$ и $y^{(\alpha+1)}$, $\alpha=1, \ldots, p-1$ :

$$
Z_{N}=\int \prod_{\alpha=1}^{p} \varrho_{\alpha}\left(y^{(\alpha)}, x^{(\alpha)}\right) \prod_{\alpha=1}^{p-1} d \mu_{\alpha}\left(x^{(\alpha)}, y^{(\alpha+1)}\right)
$$

где

$$
\begin{aligned}
& \varrho_{1}\left(y^{(1)}, x^{(1)}\right)=\operatorname{det}\left(\left(x_{i}^{(1)}\right)^{y_{j}^{(1)}}\right)_{i, j=1, \ldots, N}=\prod_{i>j}^{N}\left(x_{i}^{(1)}-x_{j}^{(1)}\right)=: \Delta_{N}\left(x^{(1)}\right), \\
& \varrho_{p}\left(y^{(p)}, x^{(p)}\right)=\operatorname{det}\left(\left(y_{i}^{(p)}\right)^{x_{j}^{(p)}}\right)_{i, j=1, \ldots, N}=\prod_{i>j}^{N}\left(y_{i}^{(p)}-y_{j}^{(p)}\right)=: \Delta_{N}\left(y^{(p)}\right)
\end{aligned}
$$

суть определители Вандермонда и

$$
\varrho_{\alpha}\left(y_{i}^{(\alpha)}, x_{j}^{(\alpha)}\right)=\operatorname{det} \rho_{\alpha}\left(y_{i}^{(\alpha)}, x_{j}^{(\alpha)}\right)_{i, j=1, \ldots, N}, \quad \alpha=2, \ldots, p-1 .
$$

Интеграл (1.3) соответствует так называемым детерминантным ансамблям [1].

Разложим каждый определитель $\operatorname{det} \rho_{\alpha}$ в сумму $N$ ! мономов (каждый из которых соответствует элементу группы перестановок $S_{N}$ ) и произведем в каждом отдельном элементе группы перестановок, например, в $\sigma$, замену переменных внутри каждого $N$-кратного интеграла в левой части $(1.3): x^{(\beta)} \rightarrow \sigma\left(x^{(\beta)}\right), y^{(\beta)} \rightarrow \sigma\left(y^{(\beta)}\right)$ для всех $\beta \leqslant \alpha$. Тогда с учетом антисимметрии определителя $\Delta_{N}\left(x^{(1)}\right)$ (который представляет собой подынтегральное выражение для самого первого $N$-кратного интеграла в (1.3)) получим, что каждый из членов указанного разложения дает один и тот же вклад. С помощью этого стандартного приема перепишем (1.3) в виде $(N !)^{2 p-2}$-кратного интеграла:

$$
\begin{aligned}
Z_{N}=\frac{1}{(N !)^{2 p-2}} \int \cdots \int \Delta_{N}\left(x^{(1)}\right) \prod_{i=1}^{N} \rho_{2}\left(y_{i}^{(2)}, x_{i}^{(2)}\right) d \mu_{1}\left(x_{i}^{(1)}, y_{i}^{(2)}\right) \times \\
\quad \times \int \cdots \int \prod_{i=1}^{N} \rho_{3}\left(y_{i}^{(3)}, x_{i}^{(3)}\right) d \mu_{2}\left(x_{i}^{(2)}, y_{i}^{(3)}\right) \times \cdots \\
\cdots \times \int \cdots \int \prod_{i=1}^{N} \rho_{p-1}\left(y_{i}^{(p-1)}, x_{i}^{(p-1)}\right) d \mu_{p-2}\left(x_{i}^{(p-2)}, y_{i}^{(p-1)}\right) \times \\
\quad \times \int \cdots \int \prod_{i=1}^{N} d \mu_{p-1}\left(x_{i}^{(p-1)}, y_{i}^{(p)}\right) \Delta_{N}\left(y^{(p)}\right) .
\end{aligned}
$$

При специальном выборе мер $d \mu_{\alpha}$ и функций $\rho_{\alpha}$ интегралы (1.3) возникают в исследованиях некоторых многоматричных $((2 p-2)$-матричных) моделей. В этих моделях матрицы $M_{1}, M_{2}, M_{3}, \ldots, M_{2 p-4}, M_{2 p-3}, M_{2 p-2}$, собственные векторы которых совпадают с наборами $\left\{x_{i}^{(1)}\right\},\left\{y_{i}^{(2)}\right\},\left\{x_{i}^{(2)}\right\}, \ldots,\left\{y_{i}^{(p-1)}\right\},\left\{x_{i}^{(p-1)}\right\},\left\{y_{i}^{(p)}\right\}$, $i=1, \ldots, N$, соответственно, связаны в открытую цепочку. Это имеет место в 
случае, когда интегрирование по матричным элементам может быть сведено к интегрированию по собственным значениям (см. [2]-[5]) каждой из матриц. Тогда в зависимости от выбора мер $d \mu_{\alpha}$ и функций $\rho_{\alpha}$ получаются либо модели нормальных матриц, либо определенные модели случайных эрмитовых (антиэрмитовых) матриц, либо некоторые модели случайных эрмитовых (антиэрмитовых) унитарных матриц, см. работы [2], [3], [6]-[10]; описание разнообразных дискретных матричных моделей содержится в работе [3].

Например, чтобы получить статистическую сумму модели случайных эрмитовых $(N \times N)$-матриц $M_{1}, \ldots, M_{2 p-2}$, связанных в цепочку, т.е.

$$
Z_{N}=\int \exp \left(\operatorname{Tr} \sum_{k=1}^{2 p-2} V_{k}\left(M_{k}\right)+\operatorname{Tr}\left(c_{1} M_{1} M_{2}+\cdots+c_{2 p-3} M_{2 p-3} M_{2 p-2}\right)\right) \prod_{k=1}^{2 p-2} d M_{k}
$$

следует выбрать

$$
\begin{gathered}
d \mu_{\alpha}(x, y)=e^{c_{2 \alpha-1} x y+V_{2 \alpha-1}(x)+V_{2 \alpha}(y)}, \quad \alpha=1, \ldots, p-1, \\
\rho_{\alpha}(x, y)=e^{c_{2 \alpha} x y}, \quad \alpha=2, \ldots, p-1 .
\end{gathered}
$$

Тогда $x_{i}^{(\alpha)}, i=1, \ldots, N$, представляют собой собственные значения эрмитовых матриц $M_{2 \alpha-1}$ с нечетными номерами, а $y_{i}^{(\alpha)}, i=1, \ldots, N,-$ собственные значения матриц $M_{2 \alpha}, \alpha=1, \ldots, p-1$. Для удобства далее будем использовать очевидную свободу записи $d \mu_{\alpha}$ и $\rho_{\alpha}$ в виде

$$
\begin{gathered}
d \mu_{1}(x, y) \rightarrow e^{c_{1} x y+V_{1}(x)}, \quad d \mu_{p-1}(x, y) \rightarrow e^{c_{2 p-3} x y+V_{2 p-2}(x)}, \\
d \mu_{\alpha}(x, y) \rightarrow e^{c_{2 \alpha-1} x y}, \quad \alpha=2, \ldots, p-2, \\
\rho_{\alpha}(x, y)=e^{c_{2 \alpha} x y+V(x)+V(y)}, \quad \alpha=2, \ldots, p-1 .
\end{gathered}
$$

В настоящей работе мы ставим перед собой две цели.

Во-первых, мы отождествим интеграл (1.3) с фермионным вакуумным ожиданием. При этом будем использовать так называемые $p$-компонентные фермионы. Этот подход можно рассматривать как продолжение работы [8]. Во-вторых, продолжая работу [11], мы соотнесем $Z_{N}$ со связанной $p$-компонентной иерархией КадомцеваПетвиашвили (КП) или, что то же самое, с $p$-компонентной иерархией решетки Тоды $(\mathrm{PT})$. Для этого рассмотрим следующую деформацию первой и последней мер:

$$
\begin{aligned}
d \mu_{1}(x, y) \rightarrow d \mu_{1}\left(x, y \mid \mathbf{t}^{(1)}, n, \overline{\mathbf{t}}^{(1)}\right) & :=x^{n_{1}} e^{V\left(x, \mathbf{t}^{(\alpha)}\right)+V\left(x^{-1}, \overline{\mathbf{t}}^{(1)}\right)} d \mu_{1}(x, y), \\
d \mu_{p-1}(x, y) \rightarrow d \mu_{p-1}\left(x, y \mid \mathbf{t}^{(p)}, n, \overline{\mathbf{t}}^{(p)}\right) & :=y^{n_{p}} e^{V\left(y, \mathbf{t}^{(p)}\right)+V\left(y^{-1}, \overline{\mathbf{t}}^{(p)}\right)} d \mu_{p-1}(x, y),
\end{aligned}
$$

где

$$
V\left(x, \mathbf{t}^{(\alpha)}\right)=\sum_{m=1}^{\infty} x^{m} t_{m}^{(\alpha)}, \quad V\left(x^{-1}, \overline{\mathbf{t}}^{(\alpha)}\right)=\sum_{m=1}^{\infty} x^{-m} \bar{t}_{m}^{(\alpha)}, \quad \alpha=1, p,
$$

вместе со следующей деформацией функций $\rho_{\alpha}, \alpha=2, \ldots, p-1$ :

$$
\rho_{\alpha}(x, y) \rightarrow \tau_{n^{(\alpha)}}\left(\mathbf{t}^{(\alpha)}+[x], \overline{\mathbf{t}}^{(\alpha)}+[y]\right),
$$


где в правой части стоят тау-функции (пронумерованные с помощью индекса $\alpha=$ $2, \ldots, p-1)$ однокомпонентной иерархии РТ, $\mathrm{a}+[x]$ и $+[y]$ обозначают так называемый мивовский сдвиг старших времен (однокомпонентной) РТ, детали см. ниже.

Деформация (1.9)-(1.12) соотносит интегралы в (1.3) со связанными $p$-компонентными иерархиями КП. Если в выражении (1.3) взять деформированные меры и деформированные функции $\rho_{\alpha}$, как описано выше, то $Z_{N}$ становится некоторой тау-функцией связанной $p$-компонентной иерархии КП или, что то же самое, $p$-компонентной иерархии РТ, в которой комплексные числа $\mathbf{t}^{(\alpha)}=\left(t_{1}^{(\alpha)}, t_{2}^{(\alpha)}, \ldots\right)$, $\overline{\mathbf{t}}^{(\alpha)}=\left(\bar{t}_{1}^{(\alpha)}, \bar{t}_{2}^{(\alpha)}, \ldots\right)$ и целые числа $n^{(\alpha)}, \alpha=1, \ldots, p$, играют роль старших времен $p$-компонентной иерархии РТ. Для краткости будем также использовать обозначения $\mathbf{t}=\left(\mathbf{t}^{(1)}, \ldots, \mathbf{t}^{(p)}\right)$ и $\overline{\mathbf{t}}=\left(\overline{\mathbf{t}}^{(1)}, \ldots, \overline{\mathbf{t}}^{(p)}\right)$.

Следует отметить, что деформация заданная формулами (1.9)-(1.12), по-видимому, не сохраняет вид формул (1.7), (1.8). В нашем случае взаимодействие

$$
e^{c_{2 \alpha} M_{2 \alpha} M_{2 \alpha+1}+V_{2 \alpha}\left(M_{2 \alpha}\right)+V_{2 \alpha+1}\left(M_{2 \alpha+1}\right)}
$$

заменяется произвольно выбранной тау-функцией $\tau_{n^{(\alpha)}}\left(\mathbf{t}^{(\alpha)}+\left[M_{2 \alpha}\right], \overline{\mathbf{t}}^{(\alpha)}+\left[M_{2 \alpha+1}\right]\right)$ однокомпонентной иерархии РТ.

Заметим также, что мы можем получить и $N$-кратные интегралы, возникающие в одноматричной модели, если будем считать меру пропорциональной дельта-функции Дирака $\delta(x-y)$.

Данная статья представляет собой одну работу в ряду статей, посвященных фермионному подходу в многократных интегралах [12], [5]. Наши фермионные конструкции в статье [5] и в данной статье отличаются от тех, которые рассматривались в работе [8] и от той, которая рассматривалась в работе [12].

1.1. Свободные фермионы. Пусть $\mathcal{A}$ - комплексная алгебра Клиффорда над $\mathbb{C}$, порождаемая заряженными свободными фермионами $f_{i}, \bar{f}_{i}, i \in \mathbb{Z}$, удовлетворяющими коммутационным соотношениям

$$
\left[f_{i}, f_{j}\right]_{+}=\left[\bar{f}_{i}, \bar{f}_{j}\right]_{+}=0, \quad\left[f_{i}, \bar{f}_{j}\right]_{+}=\delta_{i j}
$$

Будем соотносить любой элемент линейной части

$$
W:=\left(\bigoplus_{m \in \mathbb{Z}} \mathbb{C} f_{m}\right) \oplus\left(\bigoplus_{m \in \mathbb{Z}} \mathbb{C} \bar{f}_{m}\right)
$$

со свободным фермионом. Введем также свободные фермионные поля

$$
f(x):=\sum_{k \in \mathbb{Z}} f_{k} x^{k}, \quad \bar{f}(y):=\sum_{k \in \mathbb{Z}} \bar{f}_{k} y^{-k-1},
$$

которые могут рассматриваться как производящие функции для $f_{j}, \bar{f}_{j}$.

У алгебры Клиффорда имеется следующее стандартное представление (представление Фока). Определим дополнительные и взаимно дуальные подпространства, все 
элементы которых имеют нулевую норму (относительно соответствующей квадратичной формы),

$$
W_{\mathrm{an}}:=\left(\bigoplus_{m<0} \mathbb{C} f_{m}\right) \oplus\left(\bigoplus_{m \geqslant 0} \mathbb{C} \bar{f}_{m}\right), \quad W_{\mathrm{cr}}:=\left(\bigoplus_{m \geqslant 0} \mathbb{C} f_{m}\right) \oplus\left(\bigoplus_{m<0} \mathbb{C} \bar{f}_{m}\right),
$$

и рассмотрим левый и правый $\mathcal{A}$-модули

$$
F:=\mathcal{A} / \mathcal{A} W_{\text {an }}, \quad \bar{F}:=W_{\text {cr }} \mathcal{A} \backslash \mathcal{A} .
$$

Они представляют собой циклические $\mathcal{A}$-модули, порожденные соответственно векторами

$$
|0\rangle=1 \quad \bmod \mathcal{A} W_{\text {an }}, \quad\langle 0|=1 \quad \bmod W_{\text {cr }} \mathcal{A}
$$

со следующими свойствами:

$$
\begin{array}{llll}
f_{m}|0\rangle=0, & m<0 ; & \bar{f}_{m}|0\rangle=0, & m \geqslant 0 ; \\
\langle 0| f_{m}=0, & m \geqslant 0 ; & \langle 0| \bar{f}_{m}=0, & m<0 .
\end{array}
$$

Пространства Фока $F$ и $\bar{F}$ дуальны друг другу, и их эрмитово спаривание задается линейной формой $\langle 0|\cdot| 0\rangle$ на $\mathcal{A}$, называемой вакуумным ожиданием. Она определяется формулами

$$
\begin{gathered}
\langle 0|1| 0\rangle=1 ; \quad\left\langle 0\left|f_{m} \bar{f}_{m}\right| 0\right\rangle=1, \quad m<0 ; \quad\left\langle 0\left|\bar{f}_{m} f_{m}\right| 0\right\rangle=1, \quad m \geqslant 0 ; \quad \\
\left\langle 0\left|f_{n}\right| 0\right\rangle=\left\langle 0\left|\bar{f}_{n}\right| 0\right\rangle=\left\langle 0\left|f_{m} f_{n}\right| 0\right\rangle=\left\langle 0\left|\bar{f}_{m} \bar{f}_{n}\right| 0\right\rangle=0 ; \quad\left\langle 0\left|f_{m} \bar{f}_{n}\right| 0\right\rangle=0, \quad m \neq n,
\end{gathered}
$$

причем из теоремы Вика следует, что для любого конечного множества элементов $\left\{w_{k} \in W\right\}$

$$
\begin{gathered}
\left\langle 0\left|w_{1} \ldots w_{2 n+1}\right| 0\right\rangle=0 \\
\left\langle 0\left|w_{1} \ldots w_{2 n}\right| 0\right\rangle=\sum_{\sigma \in S_{2 n}} \operatorname{sgn} \sigma\left\langle 0\left|w_{\sigma(1)} w_{\sigma(2)}\right| 0\right\rangle \ldots\left\langle 0\left|w_{\sigma(2 n-1)} w_{\sigma(2 n)}\right| 0\right\rangle,
\end{gathered}
$$

где $\sigma$ перечисляет все перестановки, для которых $\sigma(1)<\sigma(2), \ldots, \sigma(2 n-1)<\sigma(2 n)$ и $\sigma(1)<\sigma(3)<\cdots<\sigma(2 n-1)$.

Пусть теперь каждый из $\left\{w_{i}\right\}_{i=1, \ldots, N}$ представляет собой линейную комбинацию только фермионов $f_{j}, j \in \mathbb{Z}$, а каждый из $\left\{\bar{w}_{i}\right\}_{i=1, \ldots, N}-$ линейную комбинацию только фермионов $\bar{f}_{j}, j \in \mathbb{Z}$. Тогда (1.22) принимает вид

$$
\left\langle 0\left|w_{1} \ldots w_{N} \bar{w}_{N} \ldots \bar{w}_{1}\right| 0\right\rangle=\left.\operatorname{det}\left(\left\langle 0\left|w_{i} \bar{w}_{j}\right| 0\right\rangle\right)\right|_{i, j=1, \ldots, N} .
$$

В соответствии с [13], [14] для всех $N \in \mathbb{Z}$ введем состояния

$$
\langle N|:=\langle 0| C_{N},
$$

где

$$
C_{N}:= \begin{cases}\bar{f}_{0} \ldots \bar{f}_{N-1}^{(\alpha)}, & N>0 \\ 1, & N=0 \\ f_{-1} \ldots f_{N}, & N<0\end{cases}
$$


а также состояния

$$
|N\rangle:=\bar{C}_{N}|0\rangle
$$

где

$$
\bar{C}_{N}:= \begin{cases}f_{N-1} \ldots f_{0}, & N>0 \\ 1, & N=0 \\ \bar{f}_{N} \ldots \bar{f}_{-1}, & N<0 .\end{cases}
$$

Состояния (1.24) и (1.26) соответствуют левым и правым вакуумным векторам с зарядом $N$.

Далее мы будем использовать следующее обозначение:

$$
\Delta_{N}(x)= \begin{cases}\operatorname{det}\left(x_{i}^{N-k}\right)_{i, k=1, \ldots, N}, & N>0 \\ 1, & N=0 \\ 0, & N<0\end{cases}
$$

Из соотношений

$$
\left\langle 0\left|\bar{f}_{N-k} f\left(x_{i}\right)\right| 0\right\rangle=x_{i}^{N-k}, \quad\left\langle 0\left|f_{-N+k-1} \bar{f}\left(y_{i}\right)\right| 0\right\rangle=y_{i}^{N-k}, \quad k=1,2, \ldots,
$$

и равенства (1.23) следует, что

$$
\begin{aligned}
\left\langle N\left|f\left(x_{1}\right) \ldots f\left(x_{n}\right)\right| 0\right\rangle & =\delta_{n, N} \Delta_{N}(x), \\
\left\langle-N\left|\bar{f}\left(y_{1}\right) \ldots \bar{f}\left(y_{n}\right)\right| 0\right\rangle & =\delta_{n, N} \Delta_{N}(y), \quad N \in \mathbb{Z} .
\end{aligned}
$$

Следуя работам [13], [14], рассмотрим элемент из $\widehat{G L}_{\infty}$ вида

$$
g=e^{h}, \quad h=\sum_{i, j} h_{i, j} f_{i} \bar{f}_{j}, \quad h_{i, j} \in \mathbb{C} .
$$

С учетом сопряжения $(\cdot) \rightarrow g(\cdot) g^{-1}$ каждый элемент $g \in \widehat{G L}_{\infty}$ действует как линейное преобразование на пространствах $\bigoplus_{m \in \mathbb{Z}} \mathbb{C} f_{m}$ и $\bigoplus_{m \in \mathbb{Z}} \mathbb{C} \bar{f}_{m}[13],[14]$.

Предположим, что выполнено следующее условие факторизации:

$$
g=g_{+} g_{-}, \quad\left\langle 0\left|g^{+}=\left\langle 0\left|, \quad g_{-}\right| 0\right\rangle=\right| 0\right\rangle, \quad g_{+}, g_{-} \in \widehat{G L}_{\infty} .
$$

ЗАмЕчАниЕ 1.1. Хотя свойство (1.33) выполняется в весьма широком классе элементов (1.32) (который включает в себя случаи, когда сумма в (1.32) оказывается конечной), нам не известна общая теорема, фиксирующая условия, необходимые и достаточные для того, чтобы это свойство выполнялось в случае, когда сумма в выражении (1.32) бесконечна.

Рассмотрим матричный элемент

$$
\left\langle 0\left|v_{N} \ldots v_{1} g \bar{v}_{1} \ldots \bar{v}_{N}\right| 0\right\rangle,
$$

где $v_{i} \in \bigoplus_{m \in \mathbb{Z}} \mathbb{C} f_{m}$ и $\bar{v}_{i} \in \bigoplus_{m \in \mathbb{Z}} \mathbb{C} \bar{f}_{m}, i=1, \ldots, N$. Положив $w_{i}=\left(g_{+}\right)^{-1} v_{i} g_{+} \in$ $\bigoplus_{m \in \mathbb{Z}} \mathbb{C} f_{m}$ и $\bar{w}_{i}=\left(g_{-}\right) \bar{v}_{i}\left(g_{-}\right)^{-1} \in \bigoplus_{m \in \mathbb{Z}} \mathbb{C} \bar{f}_{m}$, получим

$$
\left\langle 0\left|v_{N} \ldots v_{1} g \bar{v}_{1} \ldots \bar{v}_{N}\right| 0\right\rangle=\left\langle 0\left|w_{N} \ldots w_{1} \bar{w}_{1} \ldots \bar{w}_{N}\right| 0\right\rangle=\operatorname{det}\left\langle 0\left|w_{i} \bar{w}_{j}\right| 0\right\rangle_{i, j=1, \ldots, N},
$$

где второе равенство выполняется в силу теоремы Вика (1.23). Тем самым

$$
\left\langle 0\left|v_{N} \ldots v_{1} g \bar{v}_{1} \ldots \bar{v}_{N}\right| 0\right\rangle=\operatorname{det}\left\langle 0\left|v_{i} g \bar{v}_{j} \ldots\right| 0\right\rangle_{i, j=1, \ldots, N} .
$$


1.2. Многокомпонентные фермионы. Можно получить так называемый формализм $p$-компонентных фермионов путем следующей перенумерации приведенных выше свободных фермионов и свободных фермионных полей:

$$
\begin{aligned}
f_{n}^{(\alpha)} & :=f_{p n+\alpha-1}, & \bar{f}_{n}^{(\alpha)} & :=\bar{f}_{p n+\alpha-1}, \\
f^{(\alpha)}(z) & :=\sum_{k=-\infty}^{+\infty} z^{k} f_{k}^{(\alpha)}, & \bar{f}^{(\alpha)}(z) & :=\sum_{k=-\infty}^{+\infty} z^{-k-1} \bar{f}_{k}^{(\alpha)},
\end{aligned}
$$

где $\alpha=1, \ldots, p$. Из (1.13), очевидно, имеем

$$
\left[f_{n}^{(\alpha)}, f_{m}^{(\beta)}\right]_{+}=\left[\bar{f}_{n}^{(\alpha)}, \bar{f}_{m}^{(\beta)}\right]_{+}=0, \quad\left[f_{n}^{(\alpha)}, \bar{f}_{m}^{(\beta)}\right]_{+}=\delta_{\alpha, \beta} \delta_{n, m} .
$$

Правый и левый вакуумные векторы задаются соответствующими выражениями

$$
|\underbrace{0, \ldots, 0}_{p}\rangle:=|0\rangle, \quad\langle\underbrace{0, \ldots, 0}_{p}|:=\langle 0|,
$$

где $|0\rangle$ и $\langle 0|$ введены в (1.18).

Как следует из (1.18), (1.19),

$$
\begin{aligned}
& f_{m}^{(\alpha)}|0, \ldots, 0\rangle=0, \quad m<0 ; \quad \bar{f}_{m}^{(\alpha)}|0, \ldots, 0\rangle=0, \quad m \geqslant 0 ; \\
& \langle 0, \ldots, 0| f_{m}^{(\alpha)}=0, \quad m \geqslant 0 ; \quad\langle 0, \ldots, 0| \bar{f}_{m}^{(\alpha)}=0, \quad m<0 .
\end{aligned}
$$

Введем состояние

$$
\left\langle n^{(1)}, \ldots, n^{(p)}\right|:=\langle 0, \ldots, 0| C_{n^{(1)}} \ldots C_{n^{(p)}},
$$

где

а также состояние

$$
C_{n^{(\alpha)}}:= \begin{cases}\bar{f}_{0}^{(\alpha)} \ldots \bar{f}_{n^{(\alpha)}-1}^{(\alpha)}, & n^{(\alpha)}>0 \\ 1, & n^{(\alpha)}=0 \\ f_{-1}^{(\alpha)} \ldots f_{n^{(\alpha)}}^{(\alpha)}, & n^{(\alpha)}<0\end{cases}
$$

$$
\left|n^{(1)}, \ldots, n^{(p)}\right\rangle:=\bar{C}_{n^{(p)}} \ldots \bar{C}_{n^{(1)}}|0, \ldots, 0\rangle,
$$

где

$$
\bar{C}_{n^{(\alpha)}}:= \begin{cases}f_{n^{(\alpha)}-1}^{(\alpha)} \ldots f_{0}^{(\alpha)}, & n^{(\alpha)}>0 \\ 1, & n^{(\alpha)}=0 \\ \bar{f}_{n^{(\alpha)}}^{(\alpha)} \ldots \bar{f}_{-1}^{(\alpha)}, & n^{(\alpha)}<0 .\end{cases}
$$

Будем называть (1.41) и (1.43) соответственно левым и правым заряженными вакуумными векторами с зарядом $\left(n^{(1)}, \ldots, n^{(p)}\right)$.

Легко убедиться в том, что

$$
\begin{aligned}
& f_{m}^{(\alpha)}\left|*, n^{(\alpha)}, *\right\rangle=0, \quad m<n^{(\alpha)} ; \quad \bar{f}_{m}^{(1)}\left|*, n^{(\alpha)}, *\right\rangle=0, \quad m \geqslant n^{(\alpha)} ; \\
& \left\langle *, n^{(\alpha)}, *\right| f_{m}^{(\alpha)}=0, \quad m \geqslant n^{(\alpha)} ; \quad\left\langle *, n^{(\alpha)}, *\right| \bar{f}_{m}^{(\alpha)}=0, \quad m<n^{(\alpha)},
\end{aligned}
$$

где * заменяет не относящиеся к делу компоненты вакуумного вектора. 
ЗАмЕчАНИЕ 1.2. Вычисления удобно проводить с использованием теоремы Вика в виде (1.23). Это можно сделать двумя способами. Во-первых, можно использовать (1.23), учитывая то, что $p$-компонентные фермионы состоят из обычных фермионов, см. (1.35). Во-вторых, можно применять формулу (1.23) отдельно для каждой компоненты: чтобы вычислить вакуумное ожидание оператора $O$, можно сначала представить его в виде

$$
O=\sum_{i} O_{i}^{(1)} \ldots O_{i}^{(p)}
$$

тогда

$$
\langle 0|O| 0\rangle=\sum_{i}\left\langle 0\left|O_{i}^{(1)} \ldots O_{i}^{(p)}\right| 0\right\rangle=\sum_{i}\left\langle 0\left|O_{i}^{(1)}\right| 0\right\rangle \ldots\left\langle 0\left|O_{i}^{(p)}\right| 0\right\rangle,
$$

где мы применили теорему Вика (1.23) отдельно к каждому из $\left\langle 0, \ldots, 0\left|O_{i}^{(\alpha)}\right| 0, \ldots, 0\right\rangle$.

\section{2. ФЕРМИОННОЕ ПРЕДСТАВЛЕНИЕ ДЛЯ $Z_{N}$}

Рассмотрим элемент алгебры Клиффорда следующего вида:

$$
g=e^{A_{1}} g_{2} e^{A_{2}} g_{3} \ldots e^{A_{p-2}} g_{p-1} e^{A_{p-1}},
$$

где

$$
\begin{gathered}
g_{\alpha}=e^{h_{\alpha}}, \quad h_{\alpha}=\sum_{i, j} h_{i, j}^{(\alpha)} f_{i}^{(\alpha)} \bar{f}_{j}^{(\alpha)}, \quad h_{i, j}^{(\alpha)} \in \mathbb{C} ; \\
A_{\alpha}=\iint f^{(\alpha)}(x) \bar{f}^{(\alpha+1)}(y) d \mu_{\alpha}(x, y), \quad \alpha=1, \ldots, p-1,
\end{gathered}
$$

с мерой $d \mu_{\alpha}(x, y)$, вид которой в данном случае нас не интересует. Таким образом, мы имеем

$$
f_{i}^{(\beta)} g_{\alpha}=g_{\alpha} f_{i}^{(\beta)}, \quad \alpha \neq \beta, \quad i \in \mathbb{Z}
$$

Предположим также, что каждый элемент $g_{\alpha}=e^{h^{(\alpha)}}, \alpha=2, \ldots, p-1$, можно разложить в произведение элементов $g_{\alpha}^{+}$и $g_{\alpha}^{-}$алгебры $\widehat{G L}_{\infty}^{(\alpha)}$ следующим образом:

$$
g_{\alpha}=g_{\alpha}^{+} g_{\alpha}^{-}, \quad\left\langle *, \hat{0}, *\left|g_{\alpha}^{+}=\left\langle *, \hat{0} \hat{0}, *\left|, \quad g_{\alpha}^{-}\right| *, \hat{0}, *\right\rangle=\right| *, \hat{0}, *\right\rangle,
$$

где $*$ мы вновь обозначили не относящиеся к делу компоненты вакуумного вектора (в отличие от $\alpha$-компоненты, отмеченной шляпкой).

Заметим, что по формуле (1.34)

$$
\begin{gathered}
\left\langle 0\left|\bar{f}^{(\alpha)}\left(y_{1}\right) \ldots \bar{f}^{(\alpha)}\left(y_{N}\right) g_{\alpha} f^{(\alpha)}\left(x_{N}\right) \ldots f^{(\alpha)}\left(x_{1}\right)\right| 0\right\rangle= \\
=\operatorname{det}\left\langle 0\left|\bar{f}^{(\alpha)}\left(y_{i}\right) g_{\alpha} f^{(\alpha)}\left(x_{j}\right)\right| 0\right\rangle_{i, j=1, \ldots, N}
\end{gathered}
$$

Докажем, что при специальном выборе функций $\rho_{\alpha}, \alpha=2, \ldots, p-1$, а именно при

$$
\left\langle 0\left|\bar{f}^{(\alpha)}(y) g_{\alpha} f^{(\alpha)}(x)\right| 0\right\rangle=\rho_{\alpha}(y, x)
$$


имеет место формула

$$
(N !)^{p-1}\langle N, 0, \ldots, 0,-N|g| 0,0, \ldots, 0,0\rangle=Z_{N} .
$$

В самом деле, для того чтобы получить ненулевое вакуумное ожидание в левой части последнего равенства, необходимо взять только $N$-е слагаемое $A_{1}^{N} / N$ ! в степенном ряде для $e^{A_{1}}$, поскольку $e^{A_{1}}-$ единственный множитель в $g$, содержащий первую фермионную компоненту, и матричный элемент $\left\langle N, *\left|A_{1}^{n}\right| 0, *\right\rangle=0$ при всех $n \neq N$.

Используя формулу (1.30), получим, что левая часть выражения (2.8) задается интегралом

$$
\int d \mu_{1}\left(x_{1}^{(1)}, y_{1}^{(2)}\right) \ldots \int d \mu_{1}\left(x_{N}^{(1)}, y_{N}^{(2)}\right) \Delta_{N}\left(x^{(1)}\right) R_{1}
$$

где

$$
R_{1}=\left\langle *, 0, \ldots, 0,-N\left|\bar{f}^{(2)}\left(y_{1}^{(2)}\right) \ldots \bar{f}^{(2)}\left(y_{N}^{(2)}\right) g_{2} \ldots\right| *, 0, \ldots, 0\right\rangle,
$$

звездочка на первом месте в вакуумном векторе теперь означает, что мы забываем о первой фермионной компоненте. В этом заключается первый шаг наших рассуждений.

Теперь нужно выбрать только $N$-е слагаемое $A_{2}^{N} / N$ ! в разложении следующего множителя $e^{A_{2}}$. В противном случае вакуумные ожидания второй фермионной компоненты обращаются в нуль. Это происходит из-за того, что вторая фермионная компонента присутствует только в сомножителях $e^{A_{1}}, g_{2}$ и $e^{A_{2}}$ в выражении для $g$, причем $g_{2}$ представляет собой сумму мономов, каждый из которых содержит равное число фермионов $f^{(\alpha)}$ и $\bar{f}^{(\alpha)}$, в то время как $e^{A_{1}}$ содержит только фермионы $f^{(\alpha)}$, а $e^{A_{2}}$ - только фермионы $\bar{f}^{(\alpha)}$.

Таким образом, вторая фермионная компонента дает выражение

$$
\bar{f}^{(2)}\left(y_{1}^{(2)}\right) \ldots \bar{f}^{(2)}\left(y_{N}^{(2)}\right) g_{2} f^{(2)}\left(x_{1}^{(2)}\right) \ldots f^{(2)}\left(x_{N}^{(2)}\right),
$$

которое следует проинтегрировать с мерами $\prod_{i=1}^{N} d \mu\left(*, y_{i}^{(2)}\right) d \mu\left(x_{i}^{(2)}, *\right)$, а затем подставить между $\langle N, 0, \ldots, 0,-N|$ и $|0,0, \ldots, 0,0\rangle$.

Положив

$$
\left\langle 0\left|\bar{f}^{(2)}\left(y_{1}^{(2)}\right) \ldots \bar{f}^{(2)}\left(y_{N}^{(2)}\right) g_{2} f^{(2)}\left(x_{1}^{(2)}\right) \ldots f^{(2)}\left(x_{N}^{(2)}\right)\right| 0\right\rangle=\rho_{2}\left(y^{(2)}, x^{(2)}\right)
$$

(что согласно $(2.6)$ равно $\left.\operatorname{det} \rho_{2}\left(y_{i}^{(2)}, x_{j}^{(2)}\right)\right)$, получим, что левая часть $(2.8)$ задается интегралом

$$
\begin{aligned}
\int d \mu_{1}\left(x_{1}^{(1)}, y_{1}^{(2)}\right) \ldots \int d \mu_{1}\left(x_{N}^{(1)}, y_{N}^{(2)}\right) \Delta_{N}\left(x^{(1)}\right) \times \\
\quad \times \int d \mu_{2}\left(x_{1}^{(2)}, y_{1}^{(3)}\right) \ldots \int d \mu_{2}\left(x_{N}^{(2)}, y_{N}^{(3)}\right) \operatorname{det} \rho_{3}\left(y_{i}^{(3)}, x_{j}^{(3)}\right) R_{2},
\end{aligned}
$$

где

$$
R_{2}=\left\langle *, *, 0, \ldots, 0,-N\left|\bar{f}^{(2)}\left(y_{1}^{(2)}\right) \ldots \bar{f}^{(2)}\left(y_{N}^{(2)}\right) g_{2} \ldots\right| *, *, 0, \ldots, 0\right\rangle,
$$

3 Теоретическая и математическая физика, т. 152, № 2, 2007 г. 
и звездочки стоят в вакуумном векторе на первом и втором местах, поскольку теперь мы уже забываем и о первой, и о второй фермионной компонентах.

Легко видеть, что каждую экспоненту $e^{A_{\alpha}}$ следует заменить на $N$-й член ее разложения в ряд Тейлора, так как в противном случае левая часть (2.8) обращается в нуль:

$$
\begin{aligned}
& \langle N, 0, \ldots, 0,-N|g| 0,0, \ldots, 0,0\rangle= \\
& \quad=\frac{1}{(N !)^{p-1}}\left\langle N, 0, \ldots, 0,-N\left|A_{1}^{N} g_{2} A_{2}^{N} \ldots g_{p-1} A_{p-1}^{N}\right| 0,0, \ldots, 0,0\right\rangle .
\end{aligned}
$$

Продолжая исключать шаг за шагом третью, четвертую и последующие фермионные компоненты и применяя на последнем шаге формулу (1.21), получим, что выражение (2.10) совпадает с (1.3).

В заключение сделаем следующее замечание.

ЗАмечАниЕ 2.1. Добавим в (2.1) дополнительные множители так, что

$$
\begin{gathered}
g=e^{A_{1}} g_{2} e^{A_{2}} g_{3} \ldots e^{A_{p-2}} g_{p-1} e^{A_{p-1}} \rightarrow g_{\circlearrowleft}:= \\
:=e^{A_{1}} g_{2} e^{A_{2}} g_{3} \ldots e^{A_{p-2}} g_{p-1} e^{A_{p-1}} g_{p} g_{1} e^{A_{p}},
\end{gathered}
$$

где для $\alpha=1, \ldots, p$

$$
g_{\alpha}=\exp \left(\sum_{i, j} h_{i, j}^{(\alpha)} f_{i}^{(\alpha)} \bar{f}_{j}^{(\alpha)}\right), \quad A_{\alpha}=\iint f^{(\alpha)}(x) \bar{f}^{(\alpha+1)}(y) d \mu_{\alpha}(x, y),
$$

причем $\bar{f}^{(p+1)}(y) \equiv \bar{f}^{(1)}(y)$ (таким образом, мы добавляем $g_{1}, g_{p}$ и $d \mu_{p}(x, y)$ к нашему набору данных $g_{\alpha}, \alpha=2, \ldots, p-1$, и $\left.d \mu_{\alpha}(x, y), \alpha=1, \ldots, p-1\right)$. Тогда

$$
\left\langle 0,0, \ldots, 0,0\left|g_{\circlearrowleft}\right| 0,0, \ldots, 0,0\right\rangle=\sum_{N=0}^{\infty} c_{N} Z_{N}^{\circlearrowleft},
$$

где $c_{N}$ - некоторые числа, а каждая $Z_{N}^{\circlearrowleft}$ представляет собой следующий интеграл по $2 p N$ переменным $x^{(\alpha)}=\left(x_{1}^{(\alpha)}, \ldots, x_{N}^{(\alpha)}\right)$ и $y^{(\alpha)}=\left(y_{1}^{(\alpha)}, \ldots, y_{N}^{(\alpha)}\right), \alpha=1, \ldots, p$ :

$$
Z_{N}^{\circlearrowleft}=\int \prod_{\alpha=1}^{p} \rho_{\alpha}\left(y^{(\alpha)}, x^{(\alpha)}\right) \prod_{\alpha=1}^{p} d \mu_{\alpha}\left(x^{(\alpha)}, y^{(\alpha+1)}\right), \quad y^{(p+1)} \equiv y^{(1)}
$$

(заметим, что $y^{(1)}$ и $x^{(p)}$ не определены в $(1.1)$ ).

$\mathrm{B}(2.14) d \mu_{\alpha}\left(x^{(\alpha)}, y^{(\alpha+1)}\right), \alpha=1, \ldots, p-1$, задаются с помощью соотношения $(1.2)$ и равенств

$$
d \mu_{p}\left(x^{(p)}, y^{(1)}\right):=\prod_{i=1}^{N} d \mu_{p}\left(x_{i}^{(p)}, y_{i}^{(1)}\right)
$$

а каждая $\rho_{\alpha}\left(y^{(\alpha)}, x^{(\alpha)}\right)$ определена в $(2.6),(2.7)$, где теперь $\alpha=1, \ldots, p$.

Сумма в (2.13) и ее связь с большим каноническим ансамблем замкнутых цепочек спаренных случайных матриц, а также с интегрируемыми уравнениями будет рассмотрена в следующей статье. 


\section{3. ДЕФОРМАЦИЯ МЕРЫ И СВЯЗЬ С ИНТЕГРИРУЕМЫМИ ИЕРАРХИЯМИ}

Рассмотрим описанную выше деформацию (1.9)-(1.11). Применим хорошо известное соотношение $Z_{N}=\tau_{N}\left(\mathbf{t}^{(1)}, \overline{\mathbf{t}}^{(p)}\right)$, где $\tau_{N}\left(\mathbf{t}^{(1)}, \overline{\mathbf{t}}^{(p)}\right)$ - тау-функция (однокомпонентной) иерархии РТ. В самом деле, достаточно переписать (1.3) в виде $2 N$-кратного интеграла с модифицированной мерой $d \mu^{\bmod }$ (последняя зависит от выбора $\left.\rho_{\alpha}\right)$ :

$$
\begin{aligned}
Z_{N}=\int & \prod_{i=1}^{N} d \mu^{\bmod }\left(x_{i}^{(1)}, y_{i}^{(p)}\right) x_{i}^{n_{1}} y_{i}^{n_{p}} \times \\
& \times e^{V\left(x_{i}^{-1}, \overline{\mathbf{t}}^{(1)}\right)+V\left(y_{i}^{-1}, \overline{\mathbf{t}}^{(p)}\right)} e^{V\left(x_{i}^{(1)}, \mathbf{t}^{(1)}\right)+V\left(y_{i}^{(p)}, \mathbf{t}^{(p)}\right)} \Delta_{N}\left(x^{(1)}\right) \Delta_{N}\left(y^{(p)}\right) .
\end{aligned}
$$

Более того, как функция от $\mathbf{t}^{(1)}, \mathbf{t}^{(p)}, \overline{\mathbf{t}}^{(1)}, \overline{\mathbf{t}}^{(p)}$, интеграл $Z_{N}\left(\mathbf{t}^{(1)}, \mathbf{t}^{(p)}, n_{1}, n_{p}, \overline{\mathbf{t}}^{(1)}, \overline{\mathbf{t}}^{(p)}\right)$ представляет собой тау-функцию связанной двухкомпонентной иерархии КП или, что то же самое, тау-функцию двухкомпонентной иерархии РТ, см. [1].

Рассмотрим следующие деформации функций $\rho_{\alpha}, \alpha=2, \ldots, p-1$ :

$$
\begin{aligned}
\rho_{\alpha}(x, y) \rightarrow & \rho_{\alpha}\left(x, y \mid \mathbf{t}^{(\alpha)}, n^{(\alpha)}, \overline{\mathbf{t}}^{(\alpha)}\right):= \\
:= & \left\langle n^{(\alpha)}\right| e^{H^{(\alpha)}\left(\mathbf{t}^{(\alpha)}\right)} \bar{f}^{(\alpha)}\left(y_{1}^{(\alpha)}\right) \ldots \bar{f}^{(\alpha)}\left(y_{N}^{(\alpha)}\right) \times \\
& \quad \times g_{\alpha} f^{(\alpha)}\left(x_{1}^{(\alpha)}\right) \ldots f^{(\alpha)}\left(x_{N}^{(\alpha)}\right) e^{\bar{H}^{(\alpha)}\left(\overline{\mathbf{t}}^{(\alpha)}\right)}\left|n^{(\alpha)}\right\rangle,
\end{aligned}
$$

где $\mathbf{t}^{(\alpha)}=\left(t_{1}^{(\alpha)}, t_{1}^{(\alpha)}, \ldots\right)$ и $\overline{\mathbf{t}}^{(\alpha)}=\left(\bar{t}_{1}^{(\alpha)}, \bar{t}_{2}^{(\alpha)}, \ldots\right)$ - параметры деформаций, а “гамильтонианы" $H^{(\alpha)}, \bar{H}^{(\alpha)}$ задаются соотношениями

$$
\begin{aligned}
H^{(\alpha)}\left(\mathbf{t}^{(\alpha)}\right) & =\sum_{k=1}^{\infty} H_{k}^{(\alpha)} t_{k}^{(\alpha)}, \quad \bar{H}^{(\alpha)}\left(\overline{\mathbf{t}}^{(\alpha)}\right)=\sum_{k=1}^{\infty} H_{-k}^{(\alpha)} \bar{t}_{k}^{(\alpha)}, \\
H_{k}^{(\alpha)} & =\sum_{n=-\infty}^{+\infty} f_{n}^{(\alpha)} \bar{f}_{n+k}^{(\alpha)}, \quad k= \pm 1, \pm 2, \ldots,
\end{aligned}
$$

(для удобства мы их определим для $\alpha=1, \ldots, p$ ).

Заметим, что вакуумное ожидание (3.4) по определению представляет собой тауфункцию однокомпонентной РТ [13]-[15] и в нашем случае может быть определена как

$$
\tau_{n^{(\alpha)}}\left(\mathbf{t}^{(\alpha)}+\left[x^{(\alpha)}\right], \overline{\mathbf{t}}^{(\alpha)}+\left[y^{(\alpha)}\right]\right), \quad[x]:=\left(\frac{x}{1}, \frac{x^{2}}{2}, \frac{x^{3}}{3}, \ldots\right) .
$$

Докажем теперь следующее утверждение.

УтВеРЖДЕНИЕ. Комбинация деформаций (1.7)-(1.9) и (3.1) эквивалентна замене

$$
\begin{gathered}
\langle N, 0, \ldots, 0,-N|g| 0,0, \ldots, 0,0\rangle \rightarrow \tau_{N}(\mathbf{t}, \mathbf{n}, \overline{\mathbf{t}}), \\
\tau_{N}(\mathbf{t}, \mathbf{n}, \overline{\mathbf{t}}):=\left\langle N+n^{(1)}, n^{(2)}, \ldots, n^{(p-1)},-N-n^{(p)}\right| e^{H(\mathbf{t})} g \times \\
\quad \times e^{\bar{H}(\overline{\mathbf{t}})}\left|n^{(1)}, n^{(2)}, \ldots, n^{(p-1)},-n^{(p)}\right\rangle,
\end{gathered}
$$


где

$$
H(\mathbf{t})=\sum_{\alpha=1}^{p} \sum_{k=1}^{\infty} H_{k}^{(\alpha)} t_{k}^{(\alpha)}, \quad \bar{H}(\overline{\mathbf{t}})=\sum_{\alpha=1}^{p} \sum_{k=1}^{\infty} H_{-k}^{(\alpha)} \bar{t}_{k}^{(\alpha)},
$$

a “гамильтонианы" $H_{k}^{(\alpha)}$ определены в (3.2).

ДокАзАтЕЛьство. Любой член вида (2.9), а именно, всякое выражение

$$
\begin{gathered}
\left\langle 0\left|\bar{f}^{(\alpha)}\left(y_{1}^{(\alpha)}\right) \ldots \bar{f}^{(\alpha)}\left(y_{N}^{(\alpha)}\right) g_{\alpha} f^{(\alpha)}\left(x_{1}^{(\alpha)}\right) \ldots f^{(\alpha)}\left(x_{N}^{(\alpha)}\right)\right| 0\right\rangle=\rho\left(y^{(\alpha)}, x^{(\alpha)}\right) \\
\alpha=2, \ldots, p-1
\end{gathered}
$$

теперь можно заменить выражением

$$
\begin{aligned}
& \left\langle n^{(\alpha)}\right| e^{H^{(\alpha)}\left(\mathbf{t}^{(\alpha)}\right)} \bar{f}^{(\alpha)}\left(y_{1}^{(\alpha)}\right) \ldots \bar{f}^{(\alpha)}\left(y_{N}^{(\alpha)}\right) \times \\
& \quad \times g_{\alpha} f^{(\alpha)}\left(x_{1}^{(\alpha)}\right) \ldots f^{(\alpha)}\left(x_{N}^{(\alpha)}\right) e^{\bar{H}^{(\alpha)}\left(\overline{\mathbf{t}}^{(\alpha)}\right)}\left|n^{(\alpha)}\right\rangle,
\end{aligned}
$$

которое по определению, приведенному в [13]-[15], представляет собой тау-функцию однокомпонентной РТ. В силу представления (1.34) оно равно $\operatorname{det} \rho_{\alpha}\left(x_{i}, y_{j} \mid \mathbf{t}, \mathbf{n}, \overline{\mathbf{t}}\right)$, где

$$
\rho_{\alpha}\left(x_{i}, y_{j} \mid \mathbf{t}, \mathbf{n}, \overline{\mathbf{t}}\right)=\left\langle n^{(\alpha)}\left|e^{H^{(\alpha)}\left(\mathbf{t}^{(\alpha)}\right)} \bar{f}^{(\alpha)}\left(y_{i}^{(\alpha)}\right) g_{\alpha} f^{(\alpha)}\left(x_{j}^{(\alpha)}\right) e^{\bar{H}^{(\alpha)}\left(\overline{\mathbf{t}}^{(\alpha)}\right)}\right| n^{(\alpha)}\right\rangle .
$$

В случае $\alpha=1, p$ имеем

$$
\begin{array}{r}
\left\langle N, *\left|e^{H^{(1)}\left(\mathbf{t}^{(1)}\right)} f\left(x_{1}\right) \ldots f\left(x_{N}\right) e^{\bar{H}^{(1)}\left(\overline{\mathbf{t}}^{(1)}\right)}\right| 0, *\right\rangle= \\
=a_{1} \Delta_{N}(x) e^{\sum_{i=1}^{N}\left[V\left(x_{i}, \mathbf{t}^{(1)}\right)+V\left(x_{i}^{-1}, \overline{\mathbf{t}}^{(1)}\right)\right]}, \\
\left\langle *, 0\left|e^{\bar{H}^{(p)}\left(\overline{\mathbf{t}}^{(p)}\right)} \bar{f}\left(y_{N}\right) \ldots \bar{f}\left(y_{1}\right) e^{\bar{H}^{(p)}\left(\overline{\mathbf{t}}^{(p)}\right)}\right| *,-N\right\rangle= \\
=a_{p} \Delta_{N}(y) e^{\sum_{i=1}^{N}\left[V\left(y_{i}, \mathbf{t}^{(p)}\right)+V\left(y_{i}^{-1}, \overline{\mathbf{t}}^{(p)}\right)\right]},
\end{array}
$$

где $a_{\alpha}=\exp \left(\sum_{k=1}^{\infty} k t_{k}^{(\alpha)} \bar{t}_{k}^{(\alpha)}\right)$, и мы получаем вклад в соответствующие деформации мер $d \mu_{1}$ и $d \mu_{p-1}$. Доказательство завершено.

Таким образом, мы показали, что деформации функций $\rho_{\alpha}, \alpha=2, \ldots, p-1$, и мер $d \mu_{1}, d \mu_{p-1}$ приводят к тому, что $Z_{N}$ становится равно $\tau_{N}(\mathbf{t}, \mathbf{n}, \overline{\mathbf{t}})$. Известно [13], [14], [16], что таким способом построенная $\tau_{N}(\mathbf{t}, \mathbf{n}, \overline{\mathbf{t}})$ представляет собой тауфункцию связанной $p$-компонентной иерархии КП или, эквивалентно, $p$-компонентной иерархии РТ.

\section{4. ЗАКЛЮЧЕНИЕ}

Мы установили равенство между многократным интегралом (1.3) и фермионным вакуумным ожиданием (2.8). С одной стороны, можно надеяться на то, что фермионное представление позволит вычислить разнообразные величины, связанные с матричными моделями, например, спектральные детерминанты или ряды теории возмущений (как обобщение результатов работ [12], [17]). С другой стороны, с помощью этого представления можно перевести исследование этих интегралов и связанных с ними многокомпонентных матричных моделей в область исследований многокомпонентных интегрируемых иерархий. 
Благодарности. Авторы благодарны Т. Шиоте и Я. ван де Леру за полезные обсуждения. Работа Дж. Харнада частично поддержана Natural Sciences and Engineering Research Council of Canada (NSERC), фондом FCAR du Québec. А. Ю. Орлов благодарен РФФИ (грант № 05-01-00498) и Программе Президиума РАН "Фундаментальные методы нелинейной динамики” за поддержку.

\section{Список литературы}

[1] A. Borodin, A. Soshnikov, J. Statist. Phys., 113 (2003), 595; math-ph/0212063.

[2] P. Zinn-Justin, J.-B. Zuber, J. Phys. A, 36 (2003), 3173; math-ph/0209019.

[3] A. Yu. Orlov, Internat. J. Modern Phys. A, 19:suppl2 (2004), 276; nlin.SI/0209063.

[4] A. Yu. Orlov, T. Shiota, Phys. Lett. A, 343 (2005), 384; math-ph/0501017.

[5] J. Harnad, A. Yu. Orlov, J. Phys. A, 39 (2006), 8783.

[6] M. L. Mehta, Random Matrices, 2nd ed., Academic Press, Boston, MA, 1991.

[7] C. Itzykson, J.-B. Zuber, J. Math. Phys., 21 (1980), 411.

[8] S. Kharchev, A. Marshakov, A. Mironov et al., Nucl. Phys. B, 366 (1991), 569.

[9] B. Eynard, M. L. Mehta, J. Phys. A, 31 (1998), 4449.

[10] M. Bertola, B. Eynard, J. Harnad, Commun. Math. Phys., 229 (2002), 73.

[11] A. Gerasimov, A. Marshakov, A. Mironov et al., Nucl. Phys. B, 357 (1991), 565.

[12] Дж. Харнад, А. Ю. Орлов, ТМФ, 137 (2003), 375.

[13] E. Date, M. Jimbo, M. Kashiwara, T. Miwa, "Transformation groups for soliton equations", Nonlinear Integrable Systems - Classical Theory and Quantum Theory (Kyoto, 1981), World Scientific, Singapore, 1983, 39.

[14] M. Jimbo, T. Miwa, Publ. Res. Inst. Math. Sci., 19 (1983), 943.

[15] K. Ueno, K. Takasaki, "Toda lattice hierarchy", Group Representations and Systems of Differential Equations (Tokyo, 1982), Adv. Stud. Pure Math., 4, North-Holland, Amsterdam, 1984, 1.

[16] V.G. Kac, J.W. van de Leur, "The $n$-component $K P$ hierarchy and representation theory", Important Developments in Soliton Theory, Springer Ser. Nonlinear Dynam., eds. A. S. Fokas, V. E. Zakharov, Springer, Berlin, 1993, 302.

[17] J. Harnad, A. Yu. Orlov, "Matrix integrals as Borel sums of Schur function expansions", SPT 2002: Symmetries and Perturbation Theory (Cala Gonone, 2002), eds. S. Abenda, G. Gaeta, World Scientific, River Edge, NJ, 2002, 116; nlin.SI/0209035. 